Him-Tai Tsang - Christopher J. Batie

David P. Ballou · James E. Penner-Hahn

\title{
Structural characterization of the mononuclear iron site in Pseudomonas cepacia phthalate DB01 dioxygenase using X-ray absorption spectroscopy
}

\begin{abstract}
Phthalate dioxygenase (PDO) from Pseudomonas cepacia contains a Rieske-like [2Fe-2S] cluster and a mononuclear non-heme Fe(II) site. The mononuclear iron can be replaced by a variety of divalent metal ions, although only $\mathrm{Fe}(\mathrm{II})$ permits catalytic activity. We used X-ray absorption spectroscopy to characterize the structural properties of the mononuclear iron site and to follow the structural changes in this site as a function both of Rieske site oxidation state and of phthalate binding. Data for the mononuclear site have been measured directly for PDO substituted with Co or $\mathrm{Zn}$ in the mononuclear site, and by difference for the native $3-\mathrm{Fe}$ protein. The mononuclear site was modeled well by low Z-ligation (oxygen or nitrogen) and showed no evidence for high-Z ligands (e.g., sulfur). The relatively short average first shell bond lengths and the absence of significant outer shell scattering suggest that the mononuclear site has several oxygen ligands. With $\mathrm{Zn}$ in the mononuclear site, the average bond length $(2.00 \AA)$ suggests a 5-coordinate site under all conditions. In contrast, the Co- or Fe-containing mononuclear site appeared to be 6-coordinate and changed to 5-coordinate when substrate was bound, since the first shell bond length changed from 2.08 to $2.02 \AA$ (Co) or 2.10 to $2.06 \AA(\mathrm{Fe})$. The implications of these findings for the PDO mechanism are discussed.
\end{abstract}

Key words Dioxygenase · Non-heme iron ·

$\mathrm{X}$-ray absorption spectroscopy

H.-T. Tsang · J.E. Penner-Hahn (西)

Department of Chemistry, 930 N. University Avenue,

Ann Arbor, MI 48109-1055, USA

Tel. +1-313-764-7324; fax +1-313-747-4865;

e-mail: jeph@umich.edu

C.J. Batie ${ }^{1}$ - D.P. Ballou

Department of Biological Chemistry, University of Michigan,

Ann Arbor, MI 48109-0606, USA

Current address:

${ }^{1}$ Department of Biochemistry, LSU Medical Center,

New Orleans, LA 70112, USA

\section{Introduction}

The metabolism of aromatic compounds is a topic of considerable current interest. A key class of enzyme involved in the degradation of aromatic species is comprised of the dioxygenases which are important both in catechol ring cleavage and in the conversion of aromatic compounds to catechols [1-5]. Many of the pathways in bacterial degradation of aromatic compounds involve initial dihydroxylations utilizing $\mathrm{O}_{2}$ and NADH to yield cis-dihydrodiols, followed by NAD-coupled dehydrogenations to form catechols with regeneration of NADH [6].

In Pseudomonas cepacia, the enzymes in the pathway for degradation of phthalate have been isolated and purified $[1,7,8]$. Phthalate dioxygenase (PDO) from $P$. cepacia DB01 contains both a Rieske-like [2Fe$2 \mathrm{~S}$ ] center and a mononuclear Fe(II) site and is tightly coupled to phthalate oxygenase reductase during catalytic turnover [7]. PDO is similar to other dioxygenases containing Rieske centers, including benzene dioxygenase $[9,10]$ toluene dioxygenase [11-13], benzoate 1,2dioxygenase [14], pyrazon dioxygenase [15], naphthalene dioxygenase [16, 17], and 4-methoxybenzoate$O$-demethylase [18]. In addition to the Rieske center, these oxygenases either contain a mononuclear ferrous iron as isolated or require reconstitution with $\mathrm{Fe}$ (II) for maximum activity. When $P$. cepacia DB01 is grown on phthalate as the sole carbon source, PDO constitutes ca. $5-10 \%$ of the soluble protein. This, together with the fact that PDO is remarkably stable [7], makes it ideal for studies of the general problem of biological aromatic dioxygenation.

We have previously characterized the structure of the Rieske center of phthalate dioxygenase [19-21]. In the present manuscript, we report the results of an $\mathrm{X}$ ray absorption spectroscopy (XAS) study of the mononuclear site of phthalate dioxygenase. Taken together, these results provide a substantially complete structural 
definition of the metal sites in PDO and provide insight into the catalytic mechanism of this enzyme.

\section{Materials and methods}

Sample preparation

PDO was purified from $P$. cepacia DB01 as described by Batie and Ballou [19]. The purified enzyme was depleted of its mononuclear Fe by extensive dialysis against EDTA; the dialysis buffer (0.05 M HEPES, $0.005 \mathrm{M}$ EDTA, pH 8.0) was changed three times over $24 \mathrm{~h}$. The apoenzyme was equilibrated with $0.1 \mathrm{M}$ HEPES, pH 8.0 by gel filtration and then concentrated to $4.3 \mathrm{mM}$ by ultrafiltration over Centricon-30 membranes (Amicon). Samples $(180 \mu \mathrm{l})$ were prepared by mixing PDO $(3.5 \mathrm{~m} M$ final concentration) with the following reagents, as required (final concentrations given): $\mathrm{Co}(\mathrm{II})$ and $\mathrm{Zn}(\mathrm{II}), 3.0 \mathrm{~m} M$; phthalate, $5 \mathrm{mM}$; dithionite, $2.5 \mathrm{~m} M$. Reduced samples were equilibrated with $\mathrm{N}_{2}$ prior to addition of dithionite. After a 10-min incubation, samples were frozen by immersion in liquid $\mathrm{N}_{2}$.

\section{XAS measurements}

Extended X-ray absorption fine structure (EXAFS) and X-ray absorption near edge structure (XANES) spectra were measured at the Stanford Synchrotron Radiation Laboratory (SSRL) and the National Synchrotron Light Source (NSLS, Brookhaven National Laboratory). The samples studied and the experimental conditions are summarized in Table 1. Data were measured as fluorescence excitation spectra using a large solid-angle ion chamber [22] with Ar fill gas as the X-ray fluorescence detector and a combination of Soller slits with $\mathrm{Mn}, \mathrm{Fe}$ or Ni filters (at the Fe, Co and $\mathrm{Zn}$ edges, respectively) to decrease the scattered radiation [23]. The incident and transmitted intensities were measured using $\mathrm{N}_{2}$-filled ionization chambers. Energy calibration was accomplished using an $\mathrm{Fe}$, Co or $\mathrm{Zn}$ foil as an internal standard and assigning the energy of the first inflection point of the foil absorption spectra as $7111.2 \mathrm{eV}(\mathrm{Fe}), 7709.5 \mathrm{eV}(\mathrm{Co})$ or $9660.7 \mathrm{eV}(\mathrm{Zn})$ As a measure of sample integrity, we compared the XANES spectra measured for the first and the last scan of each sample. No changes were observed over the course of data collection. For active protein ( $\mathrm{Fe}$ in the mononuclear site), no loss in activity was observed following X-ray irradiation. EXAFS data reduction followed standard procedures for background removal and normalization to a Victoreen polynomial [24]. Initial conversion from energy, $E$, to $k$-space used

$k=\sqrt{2 m_{e}\left(E-E_{0}\right) / \hbar^{2}}$

where $m_{e}$ is the electron mass and the threshold energy, $E_{0}$, was set to $7130 \mathrm{eV}(\mathrm{Fe}), 7725(\mathrm{Co})$ or $9675(\mathrm{Zn})$.

\section{Data analysis}

The EXAFS data, $\chi(k)$, were fitted to Eq. 2 using a non-linear least squares algorithm.

$\chi(k)=\sum \frac{N_{i} F_{i}(k)}{k R_{i}^{2}} \exp \left(-2 k^{2} \sigma_{i}^{2}\right) \cdot \sin \left(2 k R_{i}+\varphi_{i}(k)\right)$

In Eq. $2, N_{i}$ is the number of scatterers at distance $R_{i}$ from the absorber; $\sigma_{i}$ is the root-mean-square variation in $R_{i}$; and $F_{i}(k)$ and $\varphi_{i}(k)$ are the backscattering amplitude and total phase-shift, respectively. The subscripts $i$ refer to the $i$ th absorber-scatterer pair, and the sum is taken over all the absorber-scatterer pairs.
Table 1 Samples studied

\begin{tabular}{llllll}
\hline $\begin{array}{l}\text { Metal } \\
\text { site }^{\mathrm{a}}\end{array}$ & $\begin{array}{l}\text { Rieske } \\
\text { site }^{\mathrm{b}}\end{array}$ & $\begin{array}{l}\text { Sub- } \\
\text { strate }^{\mathrm{c}}\end{array}$ & $\begin{array}{l}\text { Beam- } \\
\text { line }^{\mathrm{d}}\end{array}$ & $\begin{array}{l}\text { Mono- } \\
\text { chromator }\end{array}$ & $\begin{array}{l}\text { Temper- } \\
\text { ature }\end{array}$ \\
\hline $\mathrm{Fe}^{\mathrm{e}}$ & Oxid. & + & $\mathrm{VII}-3$ & $\mathrm{Si}(220)$ & $10 \mathrm{~K}$ \\
$\mathrm{Fe}^{\mathrm{e}}$ & Oxid. & - & $\mathrm{X} 9-\mathrm{A}$ & $\mathrm{Si}(111)$ & $77 \mathrm{~K}$ \\
$\mathrm{Co}$ & Oxid. & + & $\mathrm{VII}-3$ & $\mathrm{Si}(400)$ & $10 \mathrm{~K}$ \\
$\mathrm{Co}^{\mathrm{f}}$ & Oxid. & \pm & II-2 & $\mathrm{Si}(220)$ & $10 \mathrm{~K}$ \\
$\mathrm{Co}^{\mathrm{f}}$ & Red. & \pm & $\mathrm{X} 11-\mathrm{A}$ & $\mathrm{Si}(111)$ & $77 \mathrm{~K}$ \\
$\mathrm{Co}^{\mathrm{g}}$ & Oxid./red. & \pm & VII-3 & $\mathrm{Si}(400)$ & $10 \mathrm{~K}$ \\
$\mathrm{Zn}$ & Oxid. & \pm & VII-3 & $\mathrm{Si}(400)$ & $10 \mathrm{~K}$ \\
$\mathrm{Zn}$ & Red. & + & VII-3 & $\mathrm{Si}(400)$ & $10 \mathrm{~K}$ \\
$\mathrm{Zn}$ & Red. & - & X11-A & $\mathrm{Si}(111)$ & $77 \mathrm{~K}$ \\
\hline
\end{tabular}

${ }^{a}$ Metal in the mononuclear site

${ }^{\mathrm{b}}$ Oxidation state of Rieske cluster: oxid. $\mathrm{Fe}(\mathrm{III}) / \mathrm{Fe}(\mathrm{III})$, red. $\mathrm{Fe}(\mathrm{II}) / \mathrm{Fe}(\mathrm{III})$

${ }^{\mathrm{c}}$ Measurements were made either in the presence $(+)$ or in the absence $(-)$ of phthalate, or both $( \pm)$

${ }^{\mathrm{d}}$ SSRL beamline VII-3 is a wiggler source, operated at $18 \mathrm{kG}$. SSRL beamline II-2 is a bending magnet source with a focusing mirror. NSLS beamlines X-11A and X-9A are bending magnet sources; $\mathrm{X}-9 \mathrm{~A}$ is equipped with a mirror for harmonic rejection. Where a mirror was not used, the monochromator was detuned by $50 \%$ for harmonic rejection

${ }^{\mathrm{e}}$ Fe present in both Rieske cluster and mononuclear site

${ }^{\mathrm{f}}$ EXAFS only

${ }^{g}$ XANES only

$\mathrm{Ab}$ initio parameters for the amplitude, $F(k)$, and phase, $\varphi(k)$ were calculated using the program FEFF 6.01a $[25,26]$. These parameters were calibrated by fitting the EXAFS data for model compounds of known structure and optimizing a $k$-independent scale factor $(S)$ and $E_{0}$ so as to give the best fit. Calibrations were based on the model compounds, $\mathrm{Co}(\mathrm{III})(\mathrm{en})_{3}$ [27] for Co-N, $\mathrm{Zn}(\mathrm{II})\left(\mathrm{H}_{2} \mathrm{O}\right)_{6}$ (dihydroxybenzoate) $)_{2} \cdot 2 \mathrm{H}_{2} \mathrm{O}$ [28] for $\mathrm{Zn}-\mathrm{O}$, $\mathrm{Fe}$ (III) (acac) $)_{3}$ [29] for $\mathrm{Fe}-\mathrm{O}$, and $\mathrm{Fe}$ (III)(SPh $)_{4}\left(\mathrm{NEt}_{4}\right)$ [30] for both $\mathrm{Fe}-\mathrm{S}$ and Co-S. ${ }^{1}$ All models were prepared following literature procedures. The average value of $S$ for these and other models was 0.9 and this was used for all subsequent curve fitting. The optimized $\Delta E_{0}$ values ( $\Delta E_{0}=$ shift in $E_{0}$ from initial value) were 10 for $\mathrm{Fe}, 6$ for $\mathrm{Co}$, and 9 for $\mathrm{Zn}$. Small uncertainties in the precise value of $E_{0}$ result in an estimated accuracy of $\pm 0.02 \AA$ in the distance determinations. For comparisons of the bond lengths of a single absorber-scatterer type, the precision is substantially better, ca. $\pm 0.01 \AA$.

In general, curve fitting to the EXAFS data used three variable parameters per shell: $N, R$ and $\sigma^{2}$. Since $N$ and $\sigma^{2}$ are highly correlated, $N$ was fixed at different chemically reasonable integer values and only $R$ and $\sigma^{2}$ were treated as freely variable parameters. Both Fourier-filtered single shells $(R=0.9-2.1 \AA$ for Fe; $R=0.9-2.2 \AA$ or $1.0-2.3 \AA$ for $\mathrm{Co} ; R=1.0-2.0 \AA$ for $\mathrm{Zn})$ and unfiltered EXAFS spectra were used for curve fitting. No significant differences in structural parameters were observed. All fits utilized $k^{3}$ weighted data over a $k$ range of $2-12 \AA^{-1}$.

For quantitative analysis of the XANES region, the data were normalized [31] by subtraction of a single low-order polynomial followed by multiplication by a single scale factor to give the best agreement, both below and above the edge, with tabulated X-ray absorption cross-sections [32]. In addition to the models discussed previously, other models used for XANES comparisons included $\mathrm{Co}(\mathrm{II})(\mathrm{en})_{3}, \quad \mathrm{Co}(\mathrm{III})\left(\mathrm{NH}_{3}\right)_{6}, \quad \mathrm{Co}(\mathrm{II})\left(\mathrm{NH}_{3}\right)_{6}, \quad \mathrm{Zn}(\mathrm{II})($ acetate $)_{2} \cdot 2 \mathrm{H}_{2} \mathrm{O}$ and $\mathrm{Zn}(\mathrm{II})$ (pyridine- $\mathrm{N}$-oxide $)_{6}$.

The $1 \mathrm{~s} \rightarrow 3 \mathrm{~d}$ transitions were isolated by subtracting the appropriate XANES background. The background was estimated by

${ }^{1}$ en Ethylenediamine, acac acetylacetonate anion, $S P h$ thiophenolate ion, $\mathrm{NEt}_{4}$ tetraethylammonium 


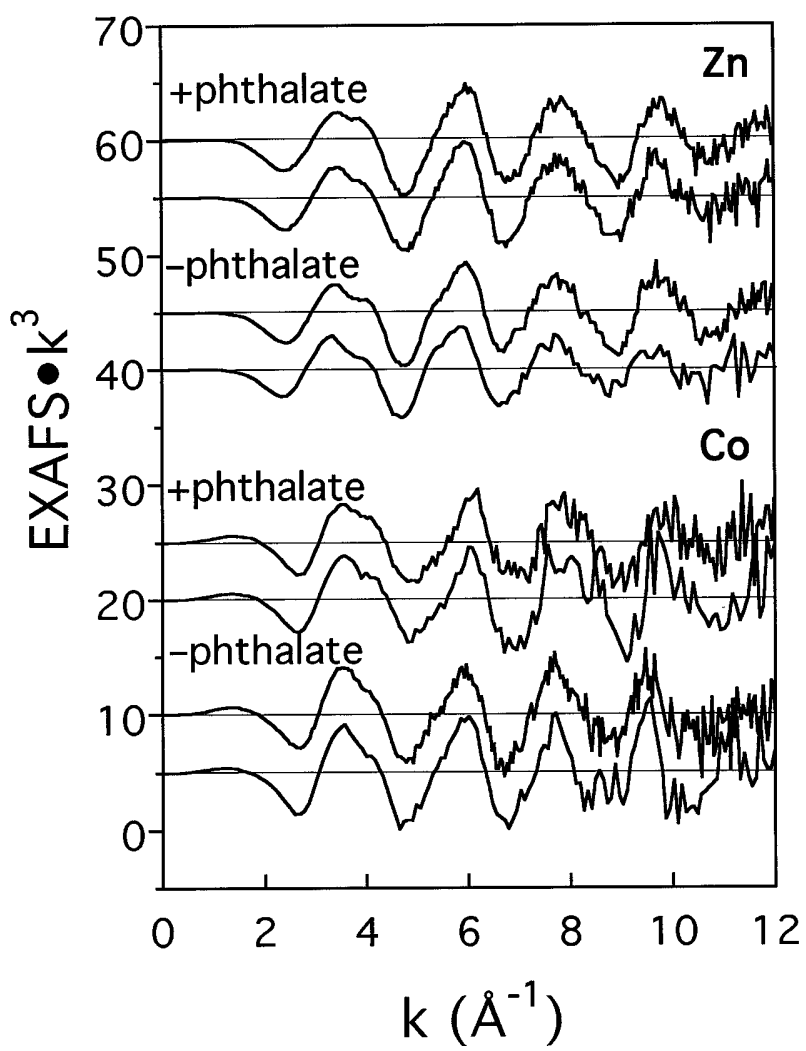

Fig. 1 EXAFS spectra ( $k^{3}$ weighted) for the mononuclear site in PDO. All spectra are plotted on the same vertical scale and displaced vertically for clarity. For each pair of spectra, the top trace is for PDO with the Rieske cluster oxidized, the bottom with the Rieske cluster reduced. From top: Zn EXAFS + phthalate; Zn EXAFS in absence of phthalate; Co EXAFS + phthalate; Co EXAFS in absence of phthalate

one of two methods: (a) fitting, in a least squares sense, a background function (linear + arctan functions) to the regions below $(7700-7707 \mathrm{eV})$ and above $(7714-7719 \mathrm{eV})$ the $1 \mathrm{~s} \rightarrow 3 \mathrm{~d}$ transition, or (b) Fitting a background function (linear + arctan) plus a Gaussian or Lorentzian function to the entire region (7700-7719 eV). Similar backgrounds were obtained using either protocol. This background was then subtracted from the data to give the isolated $1 \mathrm{~s} \rightarrow 3 \mathrm{~d}$ peak. The area of this peak was determined by numerical integration.

\section{Results}

\section{Co and Zn EXAFS}

The Co and Zn EXAFS spectra for the different Co(II) and $\mathrm{Zn}$ (II) reconstituted PDO samples are compared in Fig. 1 and the Fourier transforms of these data are compared in Fig. 2. It is clear that the gross structural features are independent of both the Rieske center oxidation state and the presence or absence of phthalate. In all cases, there is only one principal peak in the Fourier transform, centered at $R+\alpha \approx 1.5 \AA$. The data are all of reasonably high quality, although the noise level

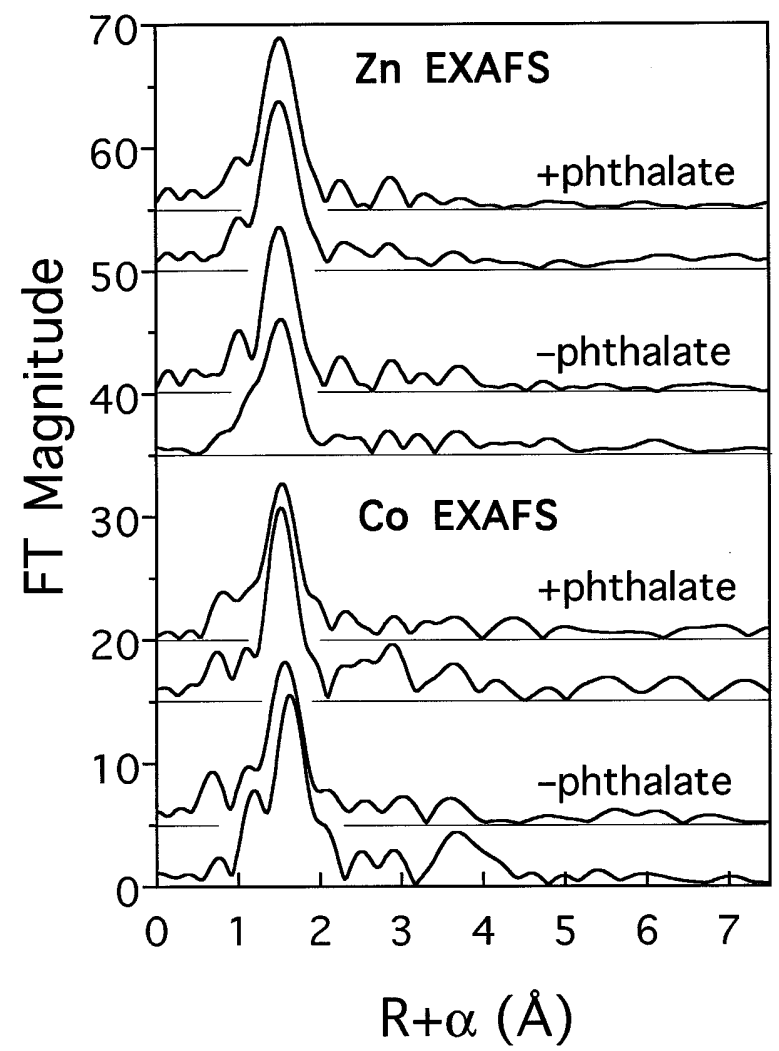

Fig. 2 Fourier transforms of the data shown in Fig. 1, calculated with $k^{3}$ weighted data over the range $2-12.5 \AA^{-1}$. All spectra are plotted on the same vertical scale and displaced for clarity. Plots are in the same order as in Fig. 1

is somewhat higher in the Co EXAFS data for the samples having reduced Rieske clusters.

On closer inspection of Figs. 1 and 2 it is apparent that the EXAFS spectra for Co-substituted PDO fall into two categories. The samples with phthalate bound have lower-frequency EXAFS, corresponding to a shorter average bond length than those without phthalate. The differences in the EXAFS can be seen most clearly in the region $k=5-7 \AA^{-1}$. In contrast to phthalate binding, changes in the oxidation state of the Rieske cluster have a much smaller effect on the Co EXAFS. Unlike Co, the EXAFS spectra for all four Zn-substituted PDO samples are quite similar, although there are, again, minor differences in the region $k=5-7 \AA^{-1}$.

The curve-fitting results for these data are given in Table 2. In all cases, the fits are consistent with the presence of only low atomic-number scatterers, i.e., nitrogen and/or oxygen. ${ }^{2}$ The spectra could not be fit using sulfur only, nor was there any significant improve-

${ }^{2}$ EXAFS amplitude and phase functions have only a weak dependence on atomic numbers; thus, it is impossible to distinguish among, for example, $\mathrm{C}, \mathrm{N}, \mathrm{O}$, and $\mathrm{F}$. Of these, the only reasonable low-atomic number scatterers are $\mathrm{N}$ and $\mathrm{O}$ 
Table 2 EXAFS curve fitting results. These results are for unfiltered data. Identical structural parameters are obtained for fits of filtered data, although $\mathrm{F}$ is smaller in this case (particularly for $\mathrm{Co}$, the F decreases ca. twofold for filtered data)

\begin{tabular}{lllllll}
\hline Metal & Rieske site $^{\mathrm{a}}$ & Phthalate $^{\mathrm{b}}$ & $\mathrm{R}$ & $\mathrm{N}^{\mathrm{c}}$ & $\sigma^{2 \mathrm{~d}}$ & $\mathrm{~F}^{\mathrm{e}}$ \\
\hline $\mathrm{Co}$ & Reduced & + & 2.02 & 3 & 1.9 & 16.0 \\
& Reduced & - & 2.08 & 4 & 3.5 & 14.5 \\
& Oxidized & + & 2.03 & 3 & 3.4 & 13.2 \\
& Oxidized & - & 2.08 & 4 & 5.2 & 12.4 \\
$\mathrm{Zn}$ & Reduced & + & 2.00 & 4 & 4.8 & 9.0 \\
& Reduced & - & 2.02 & 4 & 6.9 & 7.8 \\
& Oxidized & + & 1.99 & 4 & 4.4 & 7.2 \\
& Oxidized & - & 2.00 & 4 & 4.6 & 8.4 \\
$\mathrm{Fe}$ & Oxidized & + & 2.06 & 3 & 2.3 & 5.4 \\
& Oxidized & - & 2.10 & 2 & 0.4 & 7.6
\end{tabular}

a Oxidation state of Rieske [2Fe-2S] cluster: reduced [Fe(II)/ $\mathrm{Fe}(\mathrm{III})]$, oxidized $[\mathrm{Fe}(\mathrm{III}) / \mathrm{Fe}(\mathrm{III})]$

${ }^{\mathrm{b}}$ Sample studied in presence $(+)$ and absence $(-)$ of phthalate (see text for details)

${ }^{c}$ Fixed at integer value giving best fit using $\mathrm{M}-\mathrm{O}$ parameters

${ }^{\mathrm{d}}$ Debye-Waller factor $\times 10^{3}$ in units of $\AA^{2}$

${ }^{\mathrm{e}}$ Root-mean-square relative error defined as:

$\sqrt{\frac{\sum\left(\chi_{\mathrm{obs}}(k) \cdot k^{3}-\chi_{\mathrm{calc}}(k) \cdot k^{3}\right)^{2}}{N-1}}$

$\left[\left(\chi(k) \cdot k^{3}\right)_{\max }-\left(\chi(k) \cdot k^{3}\right)_{\min }\right]^{-1} \times 100 \%$

where $\chi_{\text {obs }}$ and $\chi_{\text {calc }}$ are the measured and fitted EXAFS amplitudes, $N$ is the number of data points, and the sum is taken over all data points. $\left(\chi(k) k^{3}\right)_{\max }$ and $\left(\chi(k) k^{3}\right)_{\min }$ are the maximum and minimum values for the weighted experimental EXAFS

ment in the fits when a shell of sulfur was added to the oxygen/nitrogen shell. For all of the EXAFS spectra, the apparent coordination numbers are surprisingly low. It is unlikely (see below) that these represent true coordination numbers of 3-4. It is more likely that the low apparent coordination numbers reflect disorder in the mononuclear site EXAFS (i.e., a range of different bond lengths).

Protein EXAFS spectra are frequently fit using two shells of scatterers, which are attributed to shorter M-O bonds and longer M-N bonds [33]. However, neither the Co nor the Zn EXAFS spectra showed evidence for two distinguishable shells of scatterers. Although there was always some improvement in the quality of the fit when the second shell is added, this improvement was small and was in no case greater than a factor of two. When the goodness of fit was normalized to the number of un-used degrees of freedom $[34,35](\approx$ eight degrees of freedom - three $(R, N, \sigma)$ or five $\left(R_{1}, R_{2}, N_{1} /\right.$ $N_{2}, \sigma_{1}, \sigma_{2}$ ) variable parameters), none of the two-shell fits were found to be significantly better than a singleshell fit. Moreover, most of the two-shell fits were characterized either by the disappearance of one of the shells $\left(\Delta \sigma^{2}\right.$ refines to $>0.02 \AA^{2}$, giving one shell an extremely small effective amplitude) or by refined bond lengths that were not resolvable with the current range of data (i.e., $\Delta R<0.15 \AA$ ). The only exception to this behavior was the Co-EXAFS for PDO with a reduced Rieske cluster and phthalate present. These data showed fourfold improvement in $F$ (ca. twofold improvement in the weighted $F$ ) when fit with shells at 2.04 and $2.19 \AA$ A. However, these data were the noisiest of the PDO spectra (see Fig. 1); thus it is unclear whether the apparent presence of two shells in this case represents a genuine structural property or merely an artifact of the high noise level of these data.

Similar conclusions have been reached from linear analyses of the EXAFS amplitudes and phases [36]. Ratios of the amplitudes for Co-substituted PDO vs. $\mathrm{Co}(\mathrm{III})\left(\mathrm{NH}_{3}\right)_{6}$ and for Zn-substituted PDO vs. $\mathrm{Zn}(\mathrm{DHB}) \cdot 6 \mathrm{H}_{2} \mathrm{O}$ were linear, demonstrating that two EXAFS-resolvable shells of scatterers do not exist in PDO.

It is important to note that these results do not imply that all of the metal-ligand distances are identical. The low apparent coordination number, or, alternatively, the large $\sigma^{2}$ for a realistic coordination number, indicated that there must have been a broad distribution of metal-ligand bond. Similar results have been found in model compounds that lack two readily distinguishable shells of scatterers [33, 37].

In favorable cases, EXAFS can provide some information regarding outer shells of scatterers. This is particularly true for rigid ligands such as imidazole, where EXAFS scattering is detectable both for the shells of carbons at ca. $3.4 \AA$ and for the $\mathrm{C} / \mathrm{N}$ shell at ca. $4.2 \AA$. The spectra in Fig. 2 show only weak contributions from outer shell scatterers. These features were at or near the noise level of the data and were not reproducible from sample to sample, suggesting that there was no significant contribution from outer shell scatterers. Since the first shell bond length was essentially identical for the Zn-substituted samples, it should be possible to average the data for these samples in order to decrease the noise level and increase the detectability of

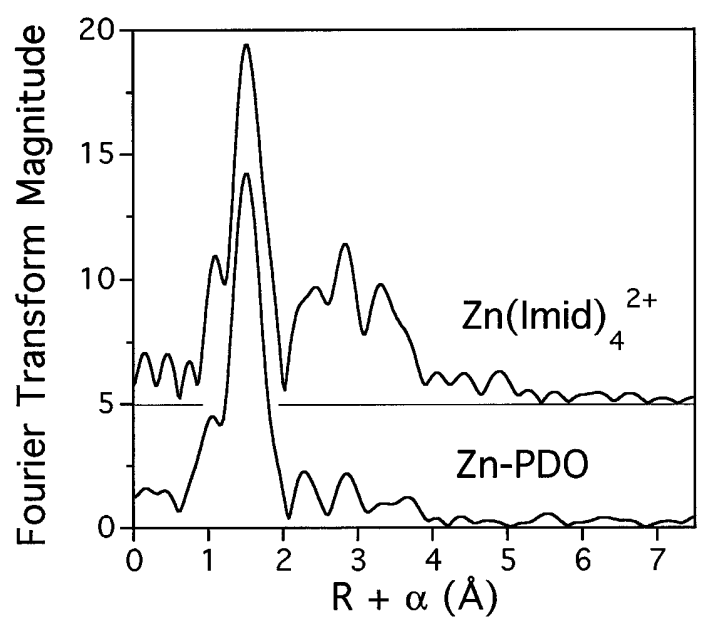

Fig. 3 Fourier transforms of the average $\mathrm{Zn}$ EXAFS for $\mathrm{Zn}$ in PDO mononuclear site and of the EXAFS for a $\mathrm{Zn}(\mathrm{His})_{4}{ }^{2+}$ model (see text). PDO data are the average of the three lower-noise spectra (SSRL data, Table 1). Fourier transform calculated using $k^{3}$ weighted data over the range $3-12.5 \AA^{-1}$. 
outer shell scattering. The Fourier transform of the average of the three lowest-noise Zn EXAFS spectra (spectra measured at SSRL, see Table 1) is shown in Fig. 3. With the lower noise level of the averaged data, weak outer shell scattering is now clearly detectable above the noise. For comparison, Fig. 3 also includes the Fourier transform for $\mathrm{Zn}(\mathrm{N} \text {-Me-imidazole })_{4}\left(\mathrm{ClO}_{4}\right)_{2}$ [38], a model having four imidazole ligands. The outer shell amplitude for $\mathrm{Zn}$ substituted PDO was roughly one-half as large as that in the model, suggesting that there are approximately two imidazole ligands. Consistent with this, fits to the outer shell scattering using FEFF 6.01a multiple scattering calculations [39] gave the best fit using approximately two imidazole ligands, with the balance of the Zn EXAFS modeled by Zn-O interactions.

\section{Iron difference EXAFS}

It is possible to calculate the EXAFS for Fe in the mononuclear site by subtracting the EXAFS for the Rieske site from the EXAFS for the native, 3-Fe protein. We have shown previously that the EXAFS for the Rieske site is independent of the occupancy of the mononuclear site and depends only on the oxidation state of the Rieske cluster [21]. Since several different measured spectra are available for the Rieske cluster, it is possible to calculate several different estimates of the mononuclear Fe EXAFS. In principle, these should be identical. In practice, the differences in the calculated mononuclear site spectra provide a measure of the uncertainty in the difference calculation.

Difference Fe EXAFS spectra were calculated for the oxidized $3-\mathrm{Fe}$ protein without phthalate minus oxidized $2-\mathrm{Fe}$ protein and for the oxidized $3-\mathrm{Fe}$ protein

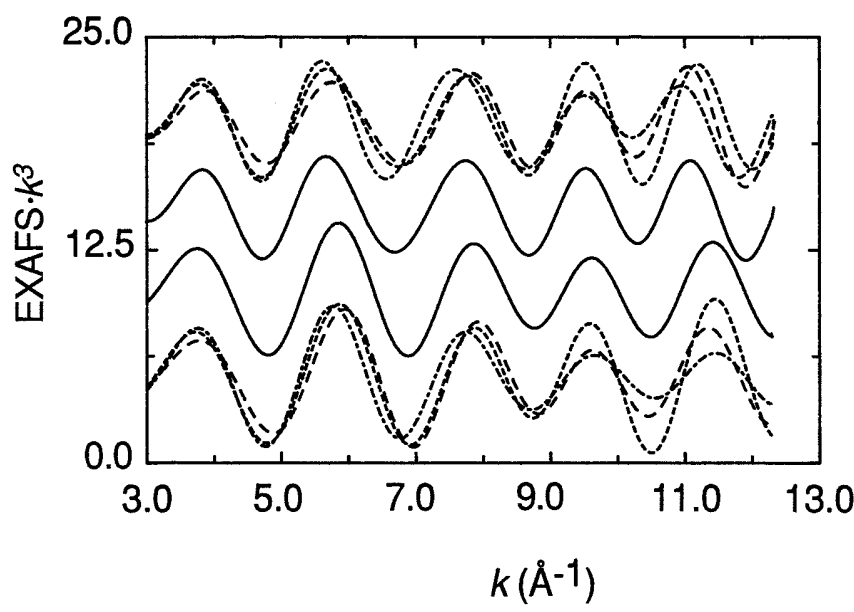

Fig. 4 Calculated Fe mononuclear site EXAFS for PDO. Calculated as difference of first shell filtered data. Top Oxidized $3 \mathrm{Fe}-$ phthalate protein minus oxidized $2 \mathrm{Fe}$ protein, bottom oxidized $3 \mathrm{Fe}+$ phthalate protein minus oxidized $2 \mathrm{Fe}$ protein. The individual difference spectra are shown as dashed lines. The average of the three difference spectra is shown as a solid line with phthalate minus oxidized 2-Fe protein. Reference spectra for the oxidized Rieske cluster were taken from data for the 2-Fe protein $+\mathrm{Zn}+$ phthalate, the 2-Fe protein + phthalate, and the $2-\mathrm{Fe}+\mathrm{Zn}$ samples, giving a total of six difference spectra. All differences were calculated using the filtered first-shell data. As shown in Fig. 4, the calculated mononuclear Fe site spectra were all very similar for $k \leq 8 \AA^{-1}$, but showed significant differences at higher $k$. These differences reflected the fact that the mononuclear site, which contains primarily low $\mathrm{Z}$ ligands, contributed relatively little to the high- $k$ EXAFS in comparison with the contributions from $\mathrm{Fe}-\mathrm{S}$ and $\mathrm{Fe}-\mathrm{Fe}$ scattering in the Rieske cluster. The results of single-shell fits to the difference EXAFS data are given in Table 2. The general trend was the same as for the Co-substituted enzyme, with addition of phthalate causing a decrease in the average Fe-ligand bond length. In view of the high noise level of these data, no attempt was made to fit the difference EXAFS spectra with multiple shells of scatterers.

\section{XANES spectra}

The normalized XANES spectra are compared in Fig. 5. The general observations were similar to those from the EXAFS. All of the spectra for the $\mathrm{Zn}$-substituted samples were quite similar. The greatest variation was observed for the spectrum for the reduced enzyme without phthalate, where all of the features were broader and less well resolved, particularly in the region $10-20 \mathrm{eV}$ above the edge. This was most likely due to the fact that this spectrum was measured under somewhat lower energy resolution conditions than were used for the other $3 \mathrm{Zn}$ XANES spectra. The spectra for the Co-substituted samples showed clear differences between the phthalate-free and the phthalate-bound forms of the enzyme. In the presence of phthalate, the main transition at $7725 \mathrm{eV}(0 \mathrm{eV}$ in Fig. 5) was more intense. In the absence of phthalate, the main transition was weaker and two new shoulders appeared at ca. 7717 and $7735 \mathrm{eV}(-8$ and $+10 \mathrm{eV}$ in Fig. 5). The former is shown more clearly in the first derivative spectra in Fig. 6.

In addition to the large, phthalate-dependent changes, there were small spectral differences, particularly in the height of the main peak, that depend on the oxidation state of the Rieske cluster. These differences can be seen as the small increase in slope for the reduced enzyme (Fig. 6) or as the tabulated peak heights (Table 3). These oxidation state-dependent differences, although small, appeared to be real, since (1) they were reproducible for spectra measured using different samples at different times with different instruments, and (2) the oxidation state of the Rieske cluster had a similar effect on both the Co and the $\mathrm{Zn}$ spectra.

The mononuclear Fe XANES spectra, calculated by difference, are also shown in Fig. 5. There was substan- 


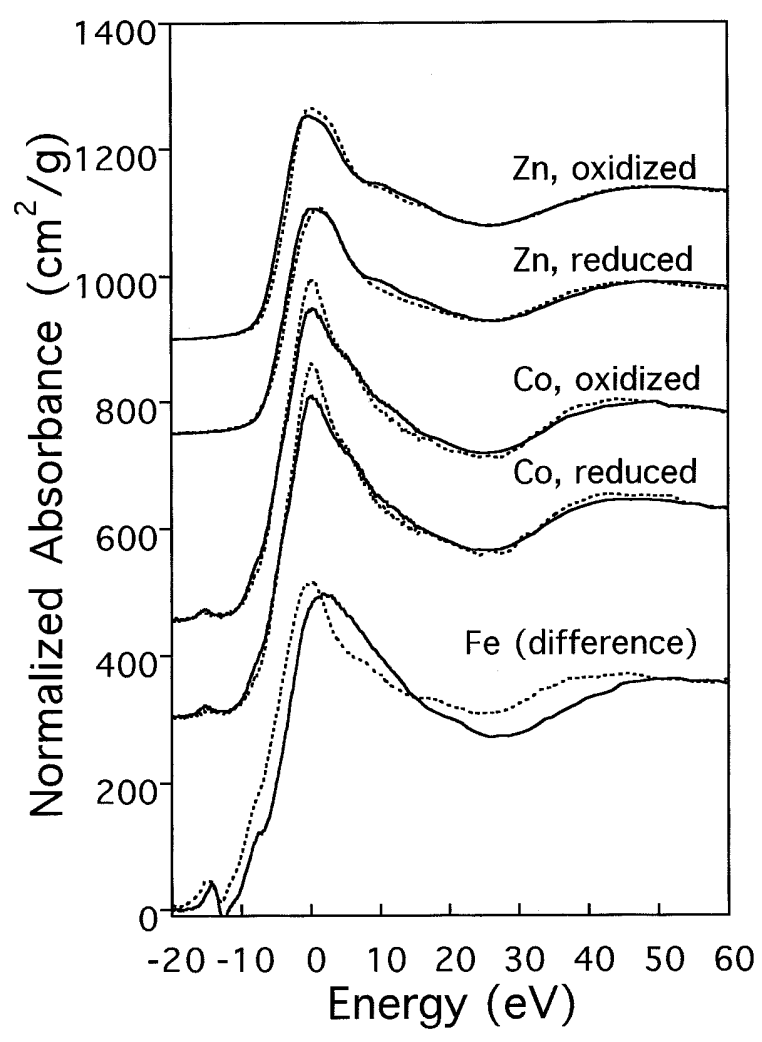

Fig. 5 Normalized XANES spectra for PDO. Top Zn, middle Co, bottom $\mathrm{Fe}$ (calculated by difference, see text). For each pair of spectra, the solid line is enzyme + phthalate, the dashed line is enzyme-phthalate. Oxidized and reduced refer to the oxidation state of the Rieske cluster. Spectra are plotted on same scale and offset vertically for clarity. Horizontal offsets are $-9668 \mathrm{eV}(\mathrm{Zn})$, $-7725 \mathrm{eV}(\mathrm{Co})$ and $-7126 \mathrm{eV}(\mathrm{Fe})$

tial uncertainty in the calculated Fe XANES spectra, as evidenced by the unusual appearance of the $1 \mathrm{~s} \rightarrow 3 \mathrm{~d}$ transition in the calculated spectra. The difference calculation was particularly difficult for the phthalate-free enzyme, since the energy resolution for the 3-Fe spectrum (measured at NSLS) was worse than that for the Rieske-only spectra. Despite these uncertainties, the qualitative trends in the Fe XANES spectra appeared to resemble those observed for the Co XANES spectra. The calculated Fe XANES spectra for the enzyme + phthalate, which should have been the most reliable, were quite similar to those observed for both Co and $\mathrm{Zn} \mathrm{PDO}+$ phthalate.

\section{$1 \mathrm{~s} \rightarrow 3 \mathrm{~d}$ transition}

The areas of the isolated $1 \mathrm{~s} \rightarrow 3 \mathrm{~d}$ transitions for the four Co spectra are given in Table 3 . The difference calculations for the mononuclear $\mathrm{Fe}$ site were too sensitive to minor differences in normalization to give reliable estimates of the $\mathrm{Fe} 1 \mathrm{~s} \rightarrow 3 \mathrm{~d}$ areas. This reflects both the presence of half as many mononuclear $\mathrm{Fe}$ atoms as

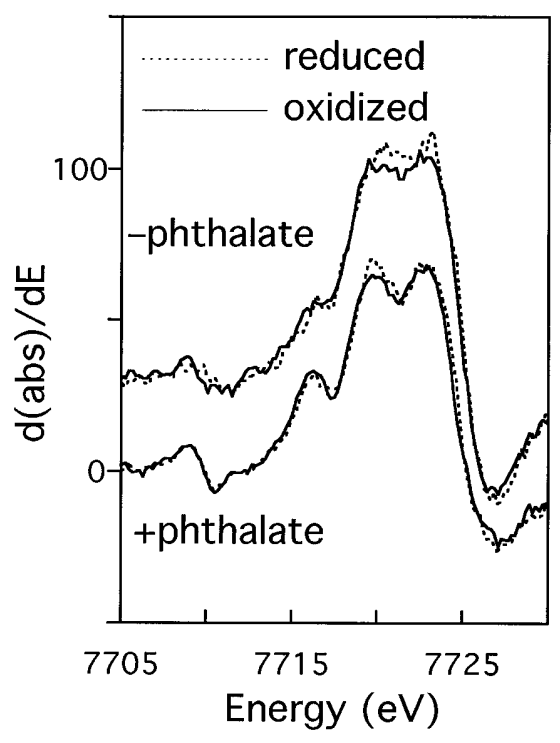

Fig. 6 First derivative with respect to energy of normalized Co XANES spectra of PDO. Top two traces (offset vertically by +30 ) are for enzyme-phthalate; bottom two are for enzyme + phthalate. For each pair of spectra, the solid line is with the Rieske cluster oxidized, the dashed line with the Rieske cluster reduced

Rieske $\mathrm{Fe}$ atoms and the fact that the $1 \mathrm{~s} \rightarrow 3 \mathrm{~d}$ transition for the mononuclear site is much weaker than that for the pseudo-tetrahedral Rieske cluster.

\section{Discussion}

Mononuclear site ligation

An inherent difficulty in using EXAFS to study PDO is the presence of multiple iron sites, which prevents direct measurement of the mononuclear site EXAFS for the intact $3-\mathrm{Fe}$ protein. The availability of numerous well-characterized mononuclear site derivatives for

Table 3 Co XANES spectra

\begin{tabular}{llcl}
\hline $\begin{array}{l}\text { Rieske } \\
\text { cluster }^{\mathrm{a}}\end{array}$ & Phthalate $^{\mathrm{b}}$ & $1 \mathrm{~s} \rightarrow 3 \mathrm{~d} \mathrm{area}^{\mathrm{c}}$ & $\begin{array}{l}\text { Principal } \\
\text { maximum }\end{array}$ \\
\hline Reduced & - & 5.8 & 540.5 \\
Oxidized & - & 7.7 & 526.7 \\
Reduced & + & 8.9 & 495.6 \\
Oxidized & + & 10.9 & 483.5 \\
\hline
\end{tabular}

${ }^{a}$ Oxidation state of Rieske cluster: reduced [Fe(II)/Fe(III)], oxidized $[\mathrm{Fe}(\mathrm{III}) / \mathrm{Fe}(\mathrm{III})]$

${ }^{b}$ Spectra measured in presence $(+)$ and absence $(-)$ of phthalate (see text for details)

${ }^{c}$ Area determined as described in text in units of $\mathrm{eV} \times 10^{-2}$; normalized to an edge jump of 1.00

${ }^{\mathrm{d}}$ Height of principal maximum in normalized XANES spectrum; spectra normalized to an edge jump from 0.0 to $331 \mathrm{~cm}^{2} / \mathrm{g}$ for Co 
PDO provides two possible solutions to this problem. For derivatives having a metal different from Fe in the mononuclear site, it is possible to probe the mononuclear site structure directly. This approach suffers from the difficulty that the structure determined may not be the same as the structure of the native mononuclear site. The alternative approach of calculating difference spectra is certain to give structural information relevant to the native mononuclear site. However, this method suffers from an inherently greater sensitivity to noise. Fortunately, in the case of PDO the two approaches yield the same result. In all cases, the data are well modeled using a single shell of low-Z scatterers with no evidence for the presence of high-Z scatterers. Although it is possible for ligands to be present in the first coordination sphere and not detectable by EXAFS, such ligands are generally distant and weakly bound [40]. In contrast, thiolate ligation, e.g., that in cytochrome $\mathrm{P}-450$, is readily detectable by EXAFS [41]. We conclude, therefore, that the mononuclear site in PDO does not have a tightly bound sulfur ligand.

Coordination numbers are not well defined in EXAFS due to the high degree of correlation between coordination number $(N)$ and Debye-Waller factor $\left(\sigma^{2}\right)$. The apparent coordination numbers of approximately four (Table 2) are best regarded as the lower limits of the true coordination number, since a broad range of metal-ligand distances can lead to an underestimate of the coordination number $[33,37]$. The apparent coordination numbers in Table 2 suggest that there is a decrease in coordination number when phthalate binds to the Co-substituted enzyme, but not when it binds to the $\mathrm{Zn}$-substituted enzyme.

In order to define more completely the mononuclear site structure, we compared the average metal-ligand bond lengths as determined by EXAFS with the bond lengths found in crystallographically characterized $\mathrm{Zn}$ and Co complexes. It is well known that, for a given metal, metal-nitrogen bond lengths are, on average, longer than metal-oxygen bond lengths, and that 6coordinate metal ions have larger radii than 5-coordinate metal ions [42]. Since the mononuclear-site metal is divalent, bond-valence-sum calculations [43, 44] can be used to characterize the ligation.

\section{Zinc site EXAFS}

The observed EXAFS bond lengths for the $\mathrm{Zn}$ site are too short to be consistent with a 6-coordinate structure, since a 6-coordinate $\mathrm{Zn}$ (II) is expected to have an average bond length $\geq 2.1 \AA[43,44]$. The observed bond lengths are consistent either with a 5-coordinate structure having primarily oxygen ligation or with a 4-coordinate structure having primarily nitrogen ligation. A 4-coordinate site would be consistent with the apparent Zn EXAFS coordination numbers (Table 2). However, a 4-coordinate site is inconsistent with the observed Co
$1 \mathrm{~s} \rightarrow 3 \mathrm{~d}$ intensity (see below). The EXAFS bond lengths are, therefore, most consistent with a 5-coordinate $\mathrm{Zn}$ site having substantial oxygen ligation. The EXAFS $\mathrm{Zn}-\mathrm{O}$ distances point to a $\mathrm{ZnO}_{5}$ site (predicted $\bar{R}_{\mathrm{Zn}-\mathrm{O}}=2.04 \AA$ ). However, given the accuracy of EXAFS bond lengths $(\approx \pm 0.02 \AA)$ and the uncertainties in bond-valence-sum calculations, it is not possible to rule out the presence of one or two nitrogen ligands.

The absence of strong outer shell EXAFS confirms that the $\mathrm{Zn}$ site is not coordinated primarily to imidazole ligands. These would be expected to give rise to Zn … C EXAFS at ca. $3.4 \AA$ and $4.2 \AA$ [45]. The outer shell EXAFS would be consistent with a small number of imidazole ligands (one or two), or with non-imidazole nitrogen ligation. Ligation by phenoxide, alkoxide, or carboxylate ligands from amino acid side-chains would be consistent with the EXAFS data. Based on sequence similarities [46] there appear to be several conserved tyrosines which might serve as ligands to the Fe. In addition, one or more water (or hydroxide) ligands may be coordinated. All of these possibilities would be consistent with the absence of intense outer shell EXAFS. However, the absence of phenolate $\rightarrow$ metal charge transfer transitions and the stabilization of the $\mathrm{Fe}$ in the ferrous oxidation state are probably inconsistent with tyrosine ligation.

Additional insight into the mononuclear site structure comes from the fact that when $\mathrm{Cu}$ is substituted at this site, the $\mathrm{Cu}$ EPR spectrum shows ligand hyperfine structure indicative of two or three histidine ligands (W.R. Dunham, personal communication), which are thus available for coordination to metals in the site. A working structural model consistent with the Zn-ligand bond lengths, the weak outer shell EXAFS, and the $\mathrm{Cu}$ $\mathrm{EPR}$ is a $\mathrm{ZnO}_{3-4}(\mathrm{Imid})_{2-1}$ structure, where the oxygencontaining ligands are most likely alkoxide, carboxylate or water (hydroxide).

\section{Cobalt site EXAFS}

In contrast with the $\mathrm{Zn}$ bond lengths, the $\mathrm{Co}-(\mathrm{O}, \mathrm{N})$ bond lengths are different for the four different forms of PDO. In the presence of phthalate, the EXAFS bond lengths $(2.03 \AA)$ are consistent with a 5-coordinate site. As with $\mathrm{Zn}$, there is no EXAFS evidence for a change in cobalt-ligand structure when the Rieske cluster is oxidized or reduced. This result is expected, since the Rieske cluster is probably $\geq 10 \AA$ from the mononuclear site. In the absence of phthalate, the EXAFS bond lengths are greater $(2.08 \AA)$, although again they show no dependence on the oxidation state of the Rieske cluster. The predicted [43, 44] average first-shell bond lengths in Co(II) complexes are 2.03-2.10 $\AA$ for 5 -coordinate complexes $\left(\mathrm{CoO}_{5}\right.$ and $\mathrm{CoN}_{5}$, respectively) and 2.10-2.17 $\AA$ for 6-coordinate complexes. The EXAFS bond lengths are thus most consistent with a 5coordinate site in the presence of phthalate and either a 
6-coordinate site or with a mixture of 5- and 6-coordinate sites in the absence of phthalate. Once again, the average bond lengths suggest that there are relatively few nitrogen ligands.

In principle, the observed $0.05-0.06 \AA$ increase in average first-shell bond length could be caused by a major change in coordination environment; for example, addition of a sulfur ligand or a change from $\mathrm{CoO}_{5}$ to $\mathrm{CoN}_{5}$ ligation. The former is inconsistent with the fact that the data are best fit with a single shell of low-Z ligands. The latter, while consistent with the EXAFS, is chemically unrealistic. The simplest interpretation is that phthalate binding causes the displacement of a cobalt ligand, thus converting a 6-coordinate site into a 5-coordinate site. This interpretation is supported by the Co XANES data.

\section{XANES spectra}

The $1 \mathrm{~s} \rightarrow 3 \mathrm{~d}$ transition is forbidden according to dipole selection rules. Nevertheless, due to weak quadrupolar coupling [47], it is always observed for metals having vacancies in the $3 \mathrm{~d}$ shell. The $1 \mathrm{~s} \rightarrow 3 \mathrm{~d}$ intensity is dramatically enhanced in non-centrosymmetric complexes as a result of $3 d+4 p$ orbital mixing [48]. The amount of $3 d+4 p$ mixing depends on the precise geometry of the complex. However, for most complexes the geometry is determined primarily by coordination number, ${ }^{3}$ and it is thus possible to use $1 \mathrm{~s} \rightarrow 3 \mathrm{~d}$ intensities to infer coordination number. Roe et al. [49] used this correlation to assign coordination numbers in Fe(III) complexes. They found that for Fe(III) complexes, the normalized $1 \mathrm{~s} \rightarrow 3 \mathrm{~d}$ areas (in units of $\mathrm{eV} \times 10^{-2}$ ) were 6-9 for 6 -coordinate complexes, 12-19 for 5-coordinate complexes and 23-25 for tetrahedral 4-coordinate complexes. The increase in the $\mathrm{Co}$ (II) $1 \mathrm{~s} \rightarrow 3 \mathrm{~d}$ area when phthalate is bound (Table 3 ) is consistent with a decrease in coordination number, in agreement with the EXAFS results.

In the absence of a database of Co(II) XANES spectra, it is difficult to correlate the areas in Table 3 with specific coordination numbers. As an approximation, we assume that the ratio of the $1 \mathrm{~s} \rightarrow 3 \mathrm{~d}$ area for $\mathrm{Co}(\mathrm{II})$ to that for $\mathrm{Fe}$ (III) depends only on the number of dvacancies. Although simplistic, this model predicts areas $(6$-coordinate $=3.6-5.4 ; 5$-coordinate $=7.2-11.5$; 4-coordinate $=13.8-15.0$ ) which are consistent with those observed for authentic 6-coordinate complexes (data not shown). Using these areas, the Co site appears to go from a 6-coordinate geometry for PDO having a reduced Rieske cluster with no phthalate bound to a 5-coordinate geometry for PDO having an oxidized Rieske cluster with phthalate bound.

\footnotetext{
${ }^{3}$ The notable exception to this rule is 4-coordinate complexes, where a change from square planar to tetrahedral gives a ca. sevenfold increase in $1 \mathrm{~s} \rightarrow 3 \mathrm{~d}$ intensity. For 5- and 6-coordinate complexes, such as those found in PDO, geometric distortions have a much smaller effect on $1 \mathrm{~s} \rightarrow 3 \mathrm{~d}$ intensity
}

The limitations of this simple model are illustrated by the fact that the oxidized/minus phthalate and reduced/plus phthalate spectra have intermediate $1 \mathrm{~s} \rightarrow 3 \mathrm{~d}$ intensities. Thus, if we were to rely on $1 \mathrm{~s} \rightarrow 3 \mathrm{~d}$ intensity alone, the coordination number would appear to decrease gradually from 6 to 5 as the Rieske cluster is oxidized and phthalate is bound. In contrast, the EXAFS bond lengths indicate a change from 6- to 5-coordinate when phthalate binds, with no dependence on Rieske cluster oxidation state. The most likely resolution of this conundrum lies in the fact that the $1 \mathrm{~s} \rightarrow 3 \mathrm{~d}$ intensity reflects both coordination number and geometry, while the average bond length depends primarily on coordination number. A variety of evidence (EPR, reactivity with $\mathrm{O}_{2}$, redox potentials) indicates that there are interactions between the Rieske and the mononuclear sites [50], and thus it is reasonable to propose that these interactions might affect the geometry of the mononuclear site.

In this model, oxidation of the Rieske cluster causes a distortion in the mononuclear site, with a corresponding increase in $1 \mathrm{~s} \rightarrow 3 \mathrm{~d}$ area by ca. $2 \times 10^{-2} \mathrm{eV}$, but with no significant change in average bond length. Binding of phthalate causes a decrease in coordination number from 6 to 5 , with a corresponding increase in $1 \mathrm{~s} \rightarrow 3 \mathrm{~d}$ area of ca. $3 \times 10^{-2} \mathrm{eV}$. If this model is correct, the minimal ranges for $\mathrm{Co}(\mathrm{II}) \mathrm{s} \rightarrow 3 \mathrm{~d}$ areas are 5.8-7.7 $\times 10^{-2} \mathrm{eV}$ for 6-coordinate complexes and $8.9-10.9 \times 10^{-2} \mathrm{eV}$ for 5-coordinate complexes. Additional model studies are necessary to test this prediction.

Regardless of their interpretation, the $1 \mathrm{~s} \rightarrow 3 \mathrm{~d}$ intensities clearly demonstrate that the mononuclear site is perturbed both by the binding of substrate and by the oxidation state of the Rieske cluster. The latter observation is important, since it provides further support for the conclusion that the mononuclear site "senses" the oxidation state of the Rieske cluster. Kinetic studies have shown that electron transfer from phthalate oxygenase reductase to the Rieske center of PDO is greatly enhanced (1000-fold) when the mononuclear site contains both $\mathrm{Fe}(\mathrm{II})$ and substrate [50]. Our finding that the structure of the mononuclear site depends on Rieske cluster oxidation state suggests a structural basis for the kinetic results.

The higher energy XANES features are more difficult to interpret than the $1 \mathrm{~s} \rightarrow 3 \mathrm{~d}$ intensities; however, they are useful as "fingerprints" of particular structures. In this context, it is noteworthy that all of the PDO XANES spectra (Fig. 5) are characterized by a single relatively narrow principal maximum and all lack the intense higher-energy multiple-scattering features observed for $\mathrm{Zn}$ (II) and $\mathrm{Co}(\mathrm{II})$ imidazole complexes [51]. This is consistent with our conclusion that there are relatively few imidazole ligands. The Co XANES features mirror the sample dependence of the $1 \mathrm{~s} \rightarrow 3 \mathrm{~d}$ transition. The dominant effect is a decrease in the principal maximum at $7725 \mathrm{eV}(0 \mathrm{eV}$ in Fig. 5). This is 
consistent with the changes expected on going from a 6to a 5-coordinate site [52]. ${ }^{4}$

As noted before, the features at 7717 and 7735 (-8 and $+10 \mathrm{eV}$ in Fig. 5) also change on phthalate binding. The $7735 \mathrm{eV}$ feature is a continuum resonance, and difficult to interpret quantitatively. In contrast, the $7717 \mathrm{eV}$ feature most likely represents a bound-state transition. Studies of $\mathrm{Cu}[53,54]$ and $\mathrm{Ni}$ [55] complexes have identified analogous shoulders on the rising edge that are attributed to a $1 s \rightarrow 4 p+$ shake-down transition. The intensity of such a transition is expected to increase on going from a pseudo-octahedral site to a 5-coordinate (e.g., square-pyramidal) site. In addition, the Co XANES spectra provide further evidence for a small structural rearrangement when the Rieske cluster is reduced. Both in the presence and in the absence of phthalate, there is a small increase in the height of the principal maximum when the Rieske cluster is reduced. The effect is much smaller than that seen when phthalate is added or removed; however, it is consistent with the conclusion, based on $1 \mathrm{~s} \rightarrow 3 \mathrm{~d}$ intensities, that the cobalt structure is slightly perturbed by the Rieske cluster oxidation state.

An advantage of the higher-energy XANES features over the $1 \mathrm{~s} \rightarrow 3 \mathrm{~d}$ transition is that they can be used to study $\mathrm{Zn}$. The Zn XANES spectra all have approximately the same height for the principal maximum. This is consistent with the lack of change in the $\mathrm{Zn}$ coordination number that was indicated by the EXAFS bond lengths. Moreover, all of the Zn XANES spectra exhibit the high-energy shoulder seen for the Co XANES spectra in the presence of phthalate. This is consistent with the proposal that the $\mathrm{Zn}$ samples and the $\mathrm{Co}+$ phthalate samples all contain a 5-coordinate metal site. The related $1 s \rightarrow 4 p+$ shake-down transition is not expected for the $\mathrm{d}^{10} \mathrm{Zn}$ (II) ion. There are small changes in the XANES spectra depending on the Rieske cluster oxidation state. These suggest that the Rieske cluster oxidation state may cause small changes in the $\mathrm{Zn}$-substituted mononuclear site similar to those proposed for the Co-substituted mononuclear site. However, for $\mathrm{Zn}$, these changes are too small to interpret reliably.

The $\mathrm{Zn}$ and Co EXAFS and XANES data thus present a consistent picture of a metal site having mixed oxygen/nitrogen ligation. For Co, the site can be either 5- or 6- coordinate, depending on whether phthalate is bound. In contrast, the $\mathrm{Zn}$ site appears to be always 5coordinate. This is consistent with the fact that the ligand-field stabilization energy for $\mathrm{Co}(\mathrm{II})$ will tend to favor higher coordination numbers, while for $\mathrm{Zn}$ the coordination number will be determined by other factors, such as steric repulsion. This difference in coordination number is similar to that observed by Yachan-

\footnotetext{
${ }^{4}$ The principal maximum is a continuum shape-resonance. It becomes narrower and more intense as the number of scatterers and the absorber-scatterer distance increase
}

dra et al. [51] for carbonic anhydrase. In that case, the native $(\mathrm{Zn})$ protein had a 4-coordinate site while the Co-reconstituted enzyme adopted either 4- or 5-coordinate structures, depending on the $\mathrm{pH}$.

To the extent that the $\mathrm{Zn} / \mathrm{Co}$ differences are governed by ligand-field stabilization energy, the native $\mathrm{Fe}$ site should behave similarly to the Co-substituted site. The Fe mononuclear site XANES spectra calculated by difference (Fig. 5) are consistent with this. In the absence of phthalate, the principal maximum is narrower and more intense than in the presence of phthalate, as expected for 6- and 5-coordinate sites, respectively. This, together with the EXAFS bond lengths (Table 2), suggests that the Fe site shows the same structural perturbations that are observed for the Co site. This is consistent with the recent observation of phthalate-dependent changes in the mononuclear site electronic structure, as reflected in the MCD spectra [56]. The latter were interpreted in terms of a 6- to 5-coordinate change. The present results provide direct structural data supporting this model.

\section{Structural implications}

The EXAFS and XANES data suggest that there is a decrease in coordination number on substrate binding. One possibility is that a protein ligand is displaced. An alternative is that phthalate binding at the active site alters the hydrophobicity of the mononuclear site environment, leading to loss of a bound water or a hydroxide ligand. This could happen, for example, if phthalate is bound with the 4,5-hydrogens (the location of hydroxylation) close to the Fe(II). Regardless of what ligand is lost, the change from 6- to 5-coordination frees a site for oxygen binding. In this regard, our proposed mechanism is reminiscent of the structural changes observed for cytochrome P-450 on substrate binding [57]. Our mechanism does not address the structural change that seems to occur when the Rieske site is reduced, since the present data only show that some change occurs, but do not indicate the nature of the change. This question, together with a detailed characterization of the $\mathrm{Fe}(\mathrm{II})$-phthalate interaction, is the subject of ongoing investigations.

\section{Conclusions}

The mononuclear site in PDO appears to have only low-Z $(\mathrm{N}, \mathrm{O})$ ligation. The relatively short EXAFS bond lengths and the very weak outer shell EXAFS features are most consistent with two or fewer imidazole ligands. The coordination number for $\mathrm{Zn}$ (II) is always 5 , while for $\mathrm{Co}(\mathrm{II})$ and $\mathrm{Fe}(\mathrm{II})$, the coordination number is either 6 (in the absence of phthalate) or 5 (in the presence of phthalate). In addition to phthalate, the mononuclear site is also sensitive to the oxidation state 
of the Rieske cluster. These results provide the first direct structural characterization of the mononuclear site and suggest some details of a possible mechanism by which the mononuclear site activates oxygen for insertion into phthalate.

Acknowledgements This work was supported in part by the National Institutes of Health, grants GM-30847 to J.E.P.H. and GM20877 to D.P.B. XAS measurements were made at SSRL, which is supported by the US DOE and by an NIH Research Resource, and at NSLS, which is supported by the US DOE. We wish to thank Profs. Sayers and Theil for facilitating access to beamline $\mathrm{X} 11 \mathrm{~A}$ at NSLS.

\section{References}

1. Keyser P, Pujar BG, Eaton RW, Ribbons DW (1976) Environ Health Perspect 18:159-166

2. Nozaki M (1979) Oxygenases and dioxygenases. In: [Dewar, MJS (ed)] Topics in current chemistry; Springer, Berlin Heidelberg New York, pp 145-186

3. Hayaishi O (1962) Annu Rev Biochem 31:25-46

4. Dagley S (1975) Am Sci 63:681-689

5. Que LJ (1980) Struct Bonding Berlin 40:39-72

6. Jeffrey AM, Yeh HJC, Jerina DM, Patel TR, Davey JF, Gibson DT (1975) Biochemistry 14:575-584

7. Batie CJ, LaHaie E, Ballou DP (1987) J Biol Chem $262: 1510-1518$

8. Keyser P (1976) PhD thesis, University of Miami

9. Axcell BC, Geary P (1975) Biochem J 146:173-183

10. Geary PJ, Saboowalla F, Patil D, Cammack R (1984) Biochem J 217:667-673

11. Yeh WK, Gibson DT, Liu TN (1977) Biochem Biophys Res Commun 78:401-410

12. Gibson DT, Yeh WK, Liu TN, Subramanian V (1982) Toluene dioxygenase: a multi component enzyme system from Pseudomonas putida. In: Nozaki M, Yamamoto S, Ishimuro Y, Coon MJ, Ernster L, Estabrook R (eds) Oxygenases and oxygen metabolism. Academic Press, New York

13. Zylstra GJ, Gibson DT (1989) J Biol Chem 264:14940-14946

14. Yamaguchi M, Fujisawa H (1980) J Biol Chem 255:5058-5063

15. Sauber K, Frohner C, Rosenberg G, Eberspacher J, Ligens F (1977) Eur J Biochem 74:89-97

16. Ensley BD, Gibson DT, Laborde AL (1982) J Bacteriol 149:948-954

17. Simon MJ, Osslund TD, Saunders R, Ensley BD, Suggs S, Harcourt A, Suen WC, Cruden DL, Gibson DT, Zylstra GJ (1993) Gene 127:31-37

18. Twilfer H, Bernhardt FH, Ğersonde K (1981) Eur J Biochem 119:595-602

19. Batie J, Ballou DP (1990) Methods Enzymol 188:61-70

20. Gurbiel RJ, Batie CJ, Sivaraja M, True AE, Fee JA, Hoffman BM, Ballou DP (1989) Biochemistry 28:4861-4871

21. Tsang HT, Batie CJ, Ballou DP, Penner-Hahn JE (1989) Biochemistry 28:7233-7240

22. Stern EA, Elam WT, Burke BA, Lu KQ, Heald SM (1982) Nucl Instrum Methods Phys Res 195:345-346

23. Stern EA, Heald SM (1979) Rev Sci Instrum 50:1579

24. MacGillavry CH, Rieck GD (eds) (1985) International tables for X-ray crystallography, vol III. Reidel, Boston
25. Rehr JJ, de Leon JM, Zabinsky SI, Albers RC (1991) J Am Chem Soc 113:5135-5140

26. Rehr JJ, Albers RC, Zabinsky SI (1992) Phys Rev Lett 69:3397-400

27. Iwata N, Nakatzu K, Saito Y (1969) Acta Crystallogr B25:2562-2571

28. Cariati, F, Erre, L, Micera, G, Panzanelli, A, Ciani, G, Sironi, A (1983) Inorg Chim Acta 80:57-65

29. Iball J, Morgan CH (1967) Acta Crystallogr 23:239-244

30. Millar M, Lee JF, Koch SA, Fikar R (1982) Inorg Chem 21:4105-4106

31. Waldo GS (1991) PhD thesis, University of Michigan, Ann Arbor, Mich

32. McMaster WH, Kerr Del Grande N, Mallett JH, Hubbell JH (1968) Compilation of X-ray cross sections. NTIS, UCRL50174, sect II, rev I

33. Riggs-Gelasco PJ, Stemmler TL, Penner-Hahn JE (1995) Coord Chem Rev 144:245-286

34. Stern EA (1993) Phys Rev B 48:9825-9827

35. Konigsberger DC (1993) Jpn J Appl Phys 32-2:877-878

36. Stern EA, Sayers DE, Lytle FW (1975) Phys Rev B $11: 4836-4846$

37. Baldwin MJ, Stemmler TL, Riggs-Gelasco PJ, Kirk ML, Penner-Hahn JE, Pecoraro VL (1994) J Am Chem Soc 116:11349-11356

38. Clark K, Tierney DL, Govindaswamy K, Gruff E, Kim C, Berg J, Koch S, Penner-Hahn JE (1995) (submitted for publication)

39. Rehr JJ, Albers RC, Zabinsky SI (1992) Phys Rev Lett 69:3397-3400

40. Penner-Hahn JE, Murata M, Freeman HC, Hodgson KO (1989) Inorg Chem 28:1826-1832

41. Hahn JE, Hodgson KO, Anderson LA, Dawson JH (1982) J Biol Chem 257:10934-10941

42. Shannon RD (1976) Acta Crystallogr A32:751-767

43. Thorp HH (1992) Inorg Chem 31:1585-8

44. Brown ID, Altermatt D (1985) Acta Crystallogr B41:244-247

45. Sandmark C, Branden CI (1967) Acta Chem Scand 21:993-999

46. Harayama, S, Kok, M, Neidle, EL (1992) Annu Rev Microbiol 46:565-601

47. Hahn JE, Scott RA, Hodgson KO, Donach S, Desjardins SR, Solomon EI (1982) Chem Phys Lett 88:595-598

48. Shulman RG, Yafet Y, Eisenberger P, Blumberg WE (1975) Proc Natl Acad Sci USA 72:4003-4007

49. Roe AL, Scheider DJ, Mayer RJ, Pyrz JW, Widom J, Que LJ (1984) J Am Chem Soc 106:1676-1681

50. Gassner G (1995) PhD thesis, University of Michigan, Ann Arbor, Mich

51. Yachandra V, Powers L, Spiro TG (1983) J Am Chem Soc 105:6596-6604

52. Bunker G (1984) PhD thesis, University of Washington, Seattle, Wash

53. Kosugi N, Yoikoyama T, Asakura K, Kuroda H (1984) Chem Phys 91:249-256

54. Shadle SE, Penner-Hahn JE, Schugar HJ, Hedman B, Hodgson KO, Solomon EI (1993) J Am Chem Soc 115:767-776

55. Shiemke AK, Shelnutt JA, Scott RA (1989) J Biol Chem 264:11236-11245

56. Gassner, GT, Ballou, DP, Landrum, GA, Whittaker, JE (1993) Biochemistry 32:4820-4825

57. Dawson JH, Sono M (1987) Chem Rev 87:1255-1276 